\title{
Um estudo observacional sobre a frequência, intensidade e climatologia de eventos extremos de chuva na Amazônia
}

\author{
An observational study of the frequency, intensity, and climatology of extreme \\ rainfall events over the Amazon
}

\author{
Adriane Lima Brito ${ }^{1}$, José Augusto P. Veiga ${ }^{1}$ \\ ${ }^{1}$ Universidade do Estado do Amazonas, UEA
}

\begin{abstract}
Resumo
O objetivo desse trabalho foi identificar os eventos extremos de chuva baseado na intensidade da precipitação, utilizando o indice R5d, com o intuito de determinar e classificar a frequência e a climatologia desses eventos. Para esta finalidade foram utilizados dados de totais diários de chuva derivados do produto MERGE do Instituto Nacional de Pesquisas Espaciais, para o período de 1998 a 2012, com enfoque principal na região da Amazônia brasileira. Após a identificação dos eventos extremos de chuva foi possível classificar esses eventos em: eventos extremos tipo I, tipo II e tipo III. Os resultados mostram que os três tipos de eventos extremos de chuva apresentam características distintas e são importantes para a modulação das chuvas na região de estudo. Ademais, observouse que os eventos extremos de chuva apresentam valores médios máximos sobre regiões especificas da Amazônia, como no oeste, sudoeste e sudeste do Estado do Amazonas e nordeste dos Estados do Pará e Amapá. Uma análise mais detalhada mostrou que os eventos extremos de chuva tipo I, apesar de mais frequentes, produzem menores quantidades média de chuvas na Bacia Amazônica $(34,6 \mathrm{~mm})$, quando comparados aos raros eventos extremos de chuva tipo III $(85,1 \mathrm{~mm})$.
\end{abstract}

Palavras-chave: Eventos extremos de chuva, climatologia, Amazônia.

\begin{abstract}
The aim of this study was to identify the extreme rainfall events based on precipitation intensity, with the purpose of determining and classifying the frequency of these events, as well as their climatology. For this purpose we have used data of total daily rainfall derived from MERGE product of the National Institute for Space Research, for the period 1998-2012, focusing the Brazilian Amazon region. After the identification of extreme rainfall events, it was possible to classifying these events into extreme events type I, type II and type III. It was observed that the three types of extreme rainfall have a mean maximum on specific regions of the Amazon, such as the west, southwest and southeast of the state of Amazonas and the northeastern states of Pará and Amapá states. A more detailed analysis showed that type I extreme rainfall, in spite of more frequent, yield small quantities of mean rainfall in the Amazon basin $(34,6 \mathrm{~mm})$, when compared to the rare type III extreme rainfall $(85,1 \mathrm{~mm})$.
\end{abstract}

Keywords: Extreme rainfall events, climatology, Amazon. 


\section{Introdução}

Eventos extremos podem ser definidos como grandes desvios, em relação à média, de um estado climático moderado, podendo apresentar frequência rara, ocorrendo em escalas de tempo que podem variar de dias até milênios (Sarewitz et al., 2000 e Marengo et al., 2007). Segundo Marengo (2009), os eventos extremos mais importantes para as atividades humanas são possivelmente os extremos de curto prazo, relacionados com o tempo, e os de médio prazo, relacionados com o clima, que são eventos que podem ocasionar impactos significativos, tanto sociais quanto econômicos e ecológicos. Assim os eventos extremos de curta duração têm sido considerados como os mais importantes.

Marengo et al. (2007) observaram que alguns modelos climáticos e estudos de projeções de clima para o futuro mostram que os eventos extremos de curta duração podem apresentar maior intensidade e frequência. Hegerl et al. (2004) analisaram a influência antrópica sobre extremos de temperatura e chuva para cenários futuros de mudanças climáticas. Dentre os resultados foi observado que as mudanças nos extremos de temperatura e chuva serão mais intensas na Amazônia. Todavia, Valverde e Marengo (2011) analisando uma climatologia baseada em índices de extremos de chuva sobre a bacia Amazônica, observaram que cenários futuros obtidos a partir do modelo ETA, forçados por condições iniciais e de fronteira do HadCM3 (Hadley Centre Coupled Model, version 3), indicam que haverá um aumento do índice CDD (dias consecutivos secos) e uma diminuição do índice R95p (dias muito úmidos) para a área da bacia Amazônica e, indicando que os períodos de secas (cheias) serão mais (menos) intensos no futuro. Marengo et al., (2011) analisaram as saídas dos modelos climáticos projetados pelo MOHC (Met Office Hadley Centre) e observaram que ocorrem reduções percentuais da precipitação pluviométrica na Amazônia a medida em que a temperatura media anual global aumenta, de forma que as mudanças mais acentuadas ocorrem depois do ano de 2040. Porém, pesquisas observacionais não mostraram sinais evidentes de tendências negativas na precipitação na Amazônia. Desta forma, considerando as incertezas existentes nos modelos climáticos, faz-se necessário um estudo dos eventos extremos de chuva usando observações do clima do presente para obter-se detalhes a respeito do comportamento desses eventos. Segundo Carvalho et al. (2004) em um futuro próximo os eventos extremos tenderão a apresentar um cenário de clima ainda mais hostil, com maiores ocorrências de estiagens e inundações. Logo, é importante utilizar uma metodologia adequada para quantificar a frequência e a intensidade de eventos extremos de chuva e quais são as suas regiões preferenciais de ocorrência.

Tendo-se em vista que há um grande interesse da comunidade científica em entender a evolução futura dos eventos extremos para planejamento e mitigação dos efeitos que eles podem causar, o presente trabalho tem por objetivo principal identificar, classificar por intensidade e estabelecer a frequência dos eventos extremos de chuva na Amazônia, assim como determinar a sua climatologia por classe de intensidade. Para esta finalidade, utilizou-se uma adaptação do critério de seleção de eventos extremos de chuva utilizado por Frich et al. (2002) e Gao et al. (2006), ver Brito (2012).

\section{Dados e Metodologia}

\section{Dados}

Os dados utilizados neste trabalho são de totais diários de chuva derivados do produto MERGE do Instituto Nacional de Pesquisas Espaciais (INPE), o qual consiste de uma técnica que combina dados de precipitação estimada pelo algoritmo 3B42RT do Tropical Rainfall Measuring Mission (TRMM) com dados de precipitação observados que são reportados em uma base de dados regular pelo Sistema Mundial de Telecomunicações, plataformas de coleta de dados e centros regionais no Brasil (Rozante et al, 2009; Vila et al, 2009). O período de estudo compreende 15 anos (1998 a 2013), onde somente os meses de dezembro, janeiro e fevereiro (DJF) são considerados. Os dados apresentam-se distribuídos em uma resolução horizontal de $20 \times 20 \mathrm{~km}$ sobre a América do Sul, no entanto o enfoque principal no que diz respeito aos resultados do trabalho é a região da Amazônia brasileira.

\section{Metodologia}

O presente trabalho utilizou uma adaptação das metodologias empregadas em Frich et al. (2002) e Gao et al. (2006) que consistem em determinar os eventos extremos de chuva (EEC) em função da média dos máximos de precipitação (MMP), R5d (precipitação máxima acumulada em 5 dias consecutivos). Neste método selecionam-se apenas os casos de máximos valores de chuva observados, por ponto de grade, em intervalos corridos de cinco dias e a partir de então se calcula a MMP de acordo com a equação abaixo

$\bar{X}_{\max }=\left(\frac{\sum X i_{\max } \bar{E}}{n}\right)$

onde, representa a MMP do período, denota os valores máximos de precipitação selecionados em cada ponto de grade e n é o número de tempos da série de dados. A partir do cálculo da MMP compara-se o valor de com cada valor de chuva do dado original de forma que: se o valor de chuva observado for igual ou maior do que este é considerado como evento extremos de chuva, caso contrário a chuva será considerada como evento normal. 
Após a identificação dos EEC calcula-se a sua média e a quantidade de chuva produzida pelos mesmos em relação ao total observado. Como próxima etapa calcula-se o desvio padrão dos máximos de precipitação (DPMP) a partir da equação abaixo,

$$
S_{\max }=\sqrt{\frac{\left(X i_{\max }-\bar{X}_{\max }\right)^{2}}{n-1}}
$$

onde, $S_{\max }$ é o DPMP.

As Figuras 1a-b, cujos valores serviram de base para a determinação dos extremos de chuva, mostram, respectivamente, a MMP e o DPMP. Observa-se na Figura 1a que na média os máximos de precipitação são localizados no oeste e sudeste do Estado do Amazonas. Os menores valores de MMP são encontrados na região nordeste do Brasil. Na Figura 1b nota-se que os valores
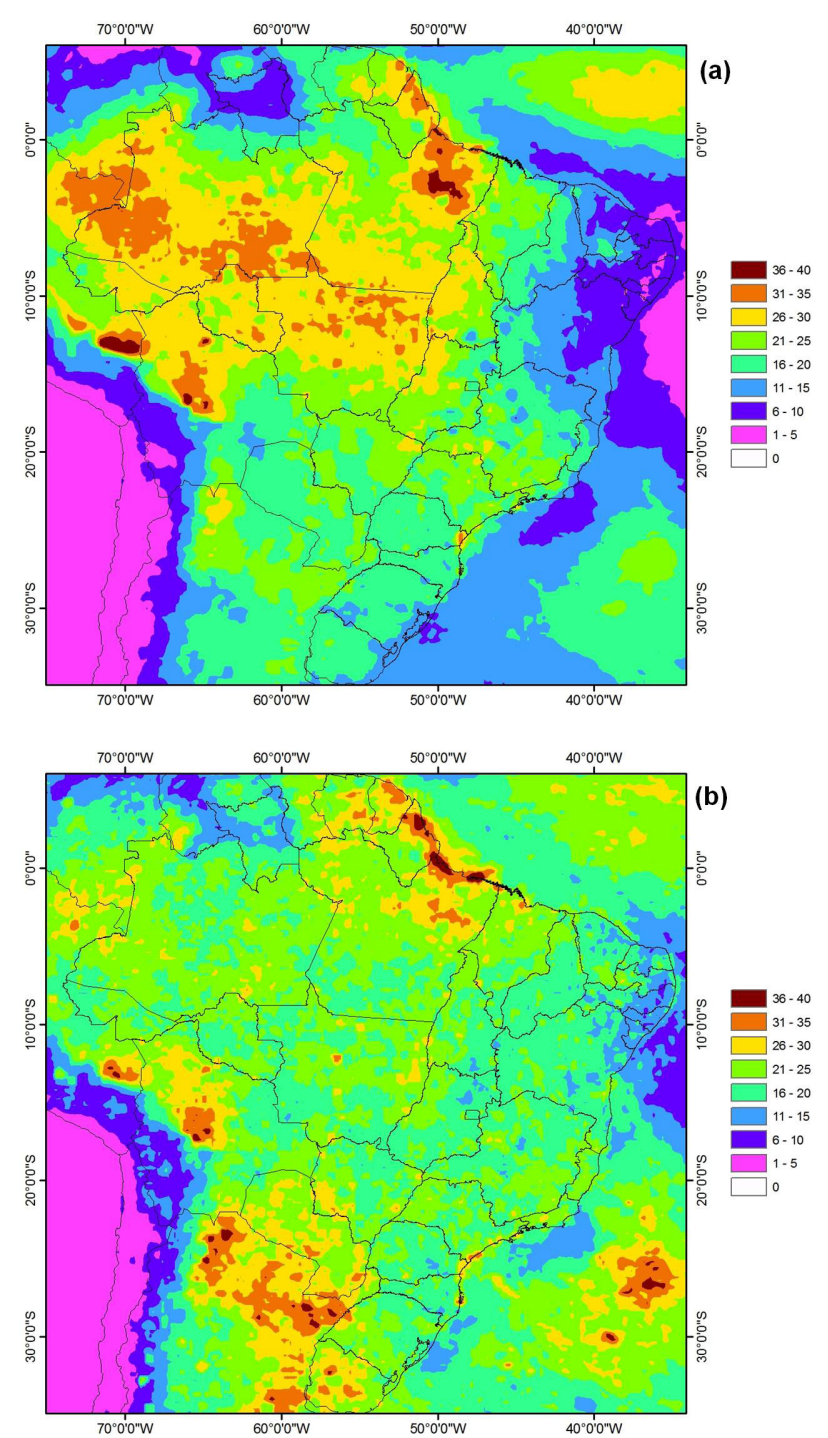

Figura 1 - (a) Média dos máximos de precipitação e (b) desvio padrão dos máximos de precipitação para DJF, obtidos a partir da Equação 1 e 2, para o período de 1998 a 2013. As unidades estão em mm máximos do DPMP ocorrem sobre a região da Amazônia brasileira com máximos sobre o nordeste do Pará e Amapá e mínimos sobre a região central de Roraima.

A partir das equações 1 e 2 é possível

classificar os EEC em função de sua intensidade. Neste método faz-se a comparação dos valores de chuva do dado original com a MMP e o DPMP.

De forma que, a classificação dos diferentes tipos de EEC é feita da seguinte maneira: caso o valor da chuva registrado no ponto de grade do domínio avaliado seja igual ou superior a MMP e ao mesmo tempo menor do que a MMP mais uma vez o desvio padrão dos máximos de precipitação (DPMP), o evento é considerado como sendo um evento extremo de chuva do tipo I (EET-I). Caso o valor da chuva registrado no ponto de grade do domínio avaliado seja igual ou superior ao da MMP mais uma vez o DPMP e ao mesmo tempo menor do que a MMP mais duas vezes o DPMP, o evento é considerado como sendo um evento extremo de chuva Tipo II (EET-II). Para que o valor de chuva seja classificado como do tipo III (EET-III), o valor da chuva registrado no ponto de grade do domínio avaliado deve ser igual ou superior ao da MMP mais duas vezes o DPMP.

\section{Resultados e discussão}

\section{a) Intensidade dos Eventos extremos}

A partir da Figura 2a é possível observar que os EET-I ocorrem sobre toda a Amazônia brasileira. De forma que, em grande parte dessa região, esse tipo de evento, produziu quantidade média de chuvas de aproximadamente $40 \mathrm{~mm}$. Os menores valores encontram-se sobre o Estado de Roraima e ficam em torno de $20 \mathrm{~mm}$. Valores máximos, em torno de $50 \mathrm{~mm}$, são observados sobre o oeste, sudoeste e sudeste do Estado do Amazonas, nordeste do Estado do Pará e nordeste do Estado do Amapá.

De acordo com a Figura 3a, os EET-II também ocorrem sobre toda a região Amazônica, apresentado valores máximos localizados nas mesmas regiões onde se encontram os máximos valores dos EET-I (oeste, sudoeste e sudeste do Estado do Amazonas, nordeste do Estado do Pará e nordeste do Estado do Amapá). No entanto, a quantidade média de chuvas produzidas pelos EET-II, nessas regiões, são maiores que a quantidade média de chuvas produzidas pelos EET-I. Observando a Figura 3a, nota-se que tipo de evento produz em média de 50 a $60 \mathrm{~mm}$ de chuva em praticamente toda a região Amazônica. Os valores máximos estão em torno de $70 \mathrm{~mm}$ sobre o oeste, sudoeste e sudeste do Estado do Amazonas, com valores próximos de $80 \mathrm{~mm}$ no nordeste dos Estados do Pará e Amapá. Os valores mínimos dos EET-II concentram-se na região do Estado de Roraima, com valores em torno de $30 \mathrm{~mm}$.

Os EET-III produziram chuvas em torno de 80 a 

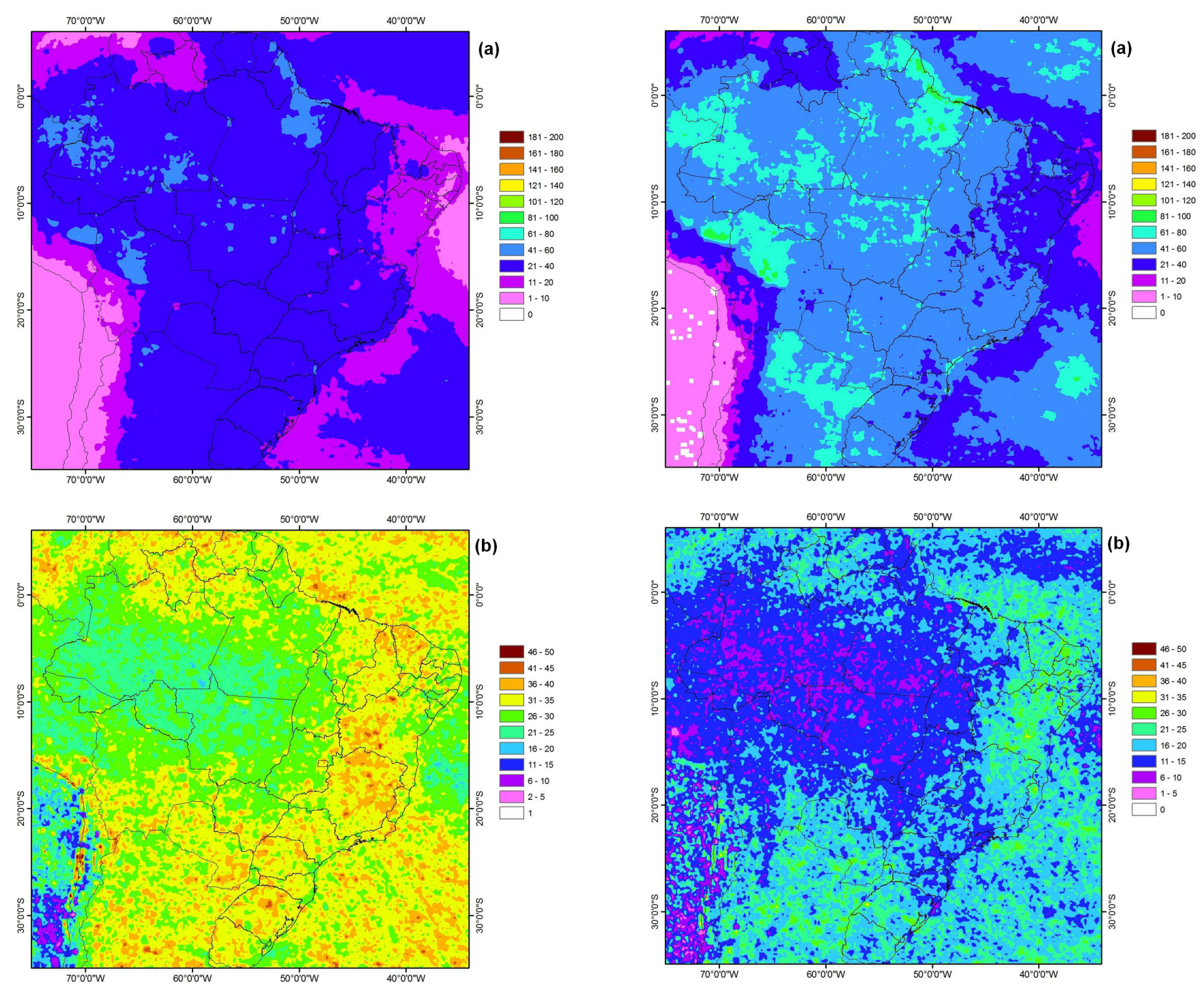

Figura 2 - (a) Quantidade de chuva média produzida pelos eventos extremos de chuva tipo I em $\mathrm{mm}$ e (b) contribuição dos eventos extremos tipo I para com o total de chuva registrado no período de 1998 a 2013 em porcentagem $\%$

$110 \mathrm{~mm}$ nessa região (Figura 4a). Apesar deste tipo de evento ocorrer em toda a Amazônia brasileira, seus valores máximos encontram-se sobre o leste e sudoeste do Estado do Amazonas (de $100 \mathrm{~mm}$ ), sobre o nordeste do estado do Amapá (120 mm) e nordeste do Pará (110 a $120 \mathrm{~mm}$ ). Os valores mínimos podem ser observados na região centro e norte do Estado do Amazonas, Sul do Pará e sobre Roraima.

A contribuição de chuva gerada pelos EET-I em relação ao total registrado no período de estudo (Figura 2b) apresenta variação de 25 a 30\% em toda a região Amazônica. Dessa forma a menor contribuição de chuva (de $25 \%$ ) gerada por esse tipo de evento ocorre na região que se estende de noroeste até sudeste da Amazônia, abrangendo uma região que vai do leste do Estado do Amazonas até o norte do Mato Grosso. Na

Figura 3 - (a) Quantidade de chuva média produzida pelos eventos extremos de chuva tipo II em $m m$ e (b) contribuição dos eventos extremos tipo II para com o total de chuva registrado no período de 1998 a 2013 em porcentagem $\%$

região norte da Amazônia a contribuição dos EET-I no total de precipitação é maior ( $30 \%)$. De acordo com a Figura $3 b$ a quantidade de chuva produzida pelos EET-II, apresenta contribuição de 10 a 15\% em relação ao total de precipitação observada no período.

De forma que, assim como na Figura 2b, os EET-II contribuem com até $10 \%$ do total de chuva em uma área que se estende do leste do Amazonas até o norte do Mato Grosso, acima dessa região (norte da Amazônia rrasileira), a contribuição desse tipo de evento aumenta para 15\%, chegando a $20 \%$ em Roraima. De acordo com a Figura $4 \mathrm{~b}$, observa-se que a quantidade de chuvas produzida pelos EET-III também possui variação em torno de 10 a $15 \%$ na região. De forma que a área de abrangência para a contribuição de $10 \%$ é maior do que o observado na figura $3 b$. Dessa forma, no extremo norte do estado 

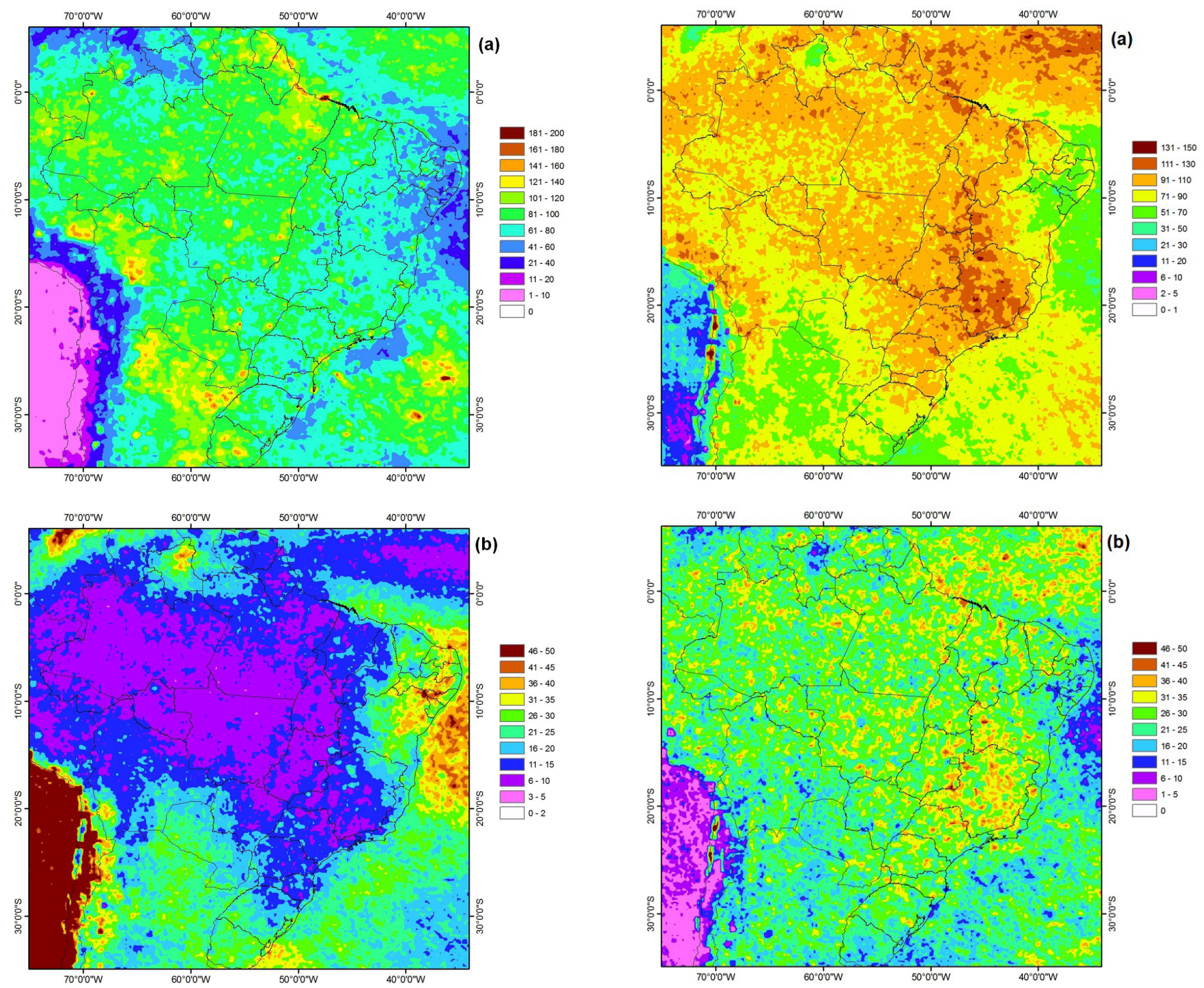

Figura 4 - (a) Quantidade de chuva média produzida pelos eventos extremos de chuva tipo III em $m m$ e (b) contribuição dos eventos extremos tipo III para com o total de chuva registrado no período de 1998 a 2013 em porcentagem $\%$

do Amazonas e no nordeste do Pará a quantidade de chuva produzida pelos EET-III possui contribuição de $15 \%$ para com o total de precipitação. No nordeste de Roraima a contribuição do EET-III chega a 30\%.

\section{b) Frequência dos Eventos extremos}

A frequência dos EET-I para o período de estudo (Figura 5a) apresenta variação de 80 a 90 casos, por ponto de grade, em grande parte da Amazônia brasileira. Mesmo estes valores não se encontrando bem distribuídos sobre a região de estudo, observa-se um maior número de casos sobre o norte da Amazônia e um menor número de casos sobre o sul da mesma região. No nordeste de Roraima, a frequência dos EET-I apresenta variação

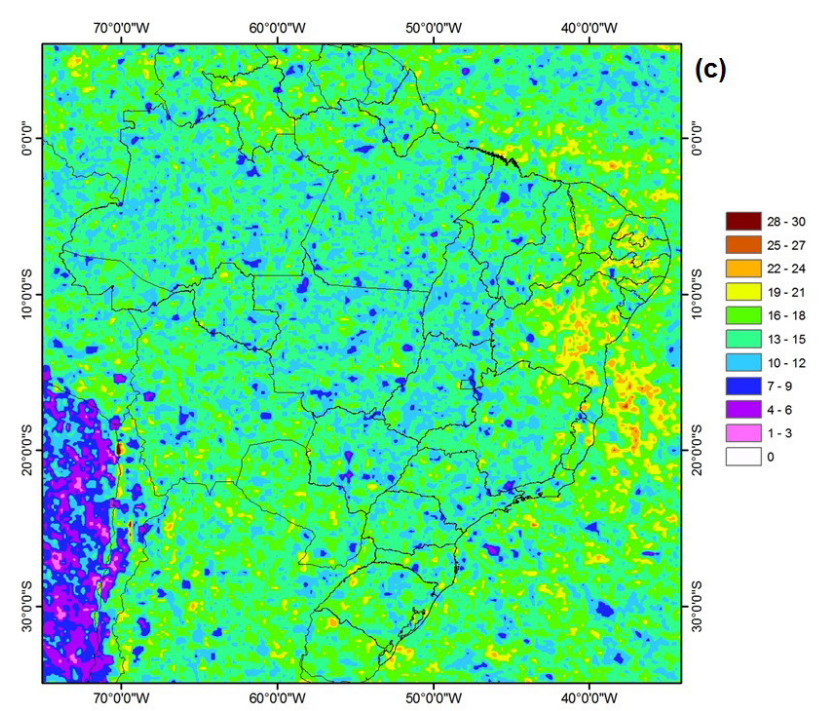

Figura 5 - Frequência dos eventos extremos tipo I (a), tipo II (b) e tipo III (c) que ocorreram por ponto de grade, dentro do período de estudo. Para isso foram utilizadas as condições para as classificações de EEC descrito anteriormente para contabilizar o número de casos de EEC 
menor do que no restante da região, ficando em torno de 40 a 70 casos. No entanto, a frequência dos EET-II apresentada na Figura 5b possui variação em torno de 20 a 40 casos. No nordeste de Roraima, a frequência dos EET-II apresenta menor número de casos ( 15).

A frequência dos EET-III (Figura 5c) também não possui distribuição bem definida sobre a região, de forma que esse tipo de evento apresenta uma frequência bem menor em relação ao que foi observado nas demais classificações, variando entre 12 a 14 casos sobre a Amazônia.

A Tabela 1 apresenta valores médios de intensidade, frequência e contribuição dos EET-I, EET-II e EET-III obtidos para a área da bacia amazônica. Nota-se que, o valor médio de chuva produzido pelos EET-III é o mais intenso, 85,1 mm, seguido do EET-II, que produz em média 55,9 mm e o EET-I com 34,6 $\mathrm{mm}$. Em contrapartida, ao se observar a frequência (número de casos por ponto de grade), nota-se que os EET-I são responsáveis pelo maior número de casos que ocorreram na região, 228 casos, seguidos pelos EET-II com 65 casos e os EET-III com 32 casos. Estes resultados revelam que quanto maior é a intensidade dos EEC, menor será a sua frequência. No que diz respeito à contribuição dos EEC para com o total de chuva observado, observa-se que os EET-I possuem maior contribuição (23,2\%), seguidos dos EET-II (9,7\%) e EET-III (7\%). Isso ocorre devido ao total de chuva produzida pelos EET-I ser muito maior do que os demais tipos de EEC.

Tabela 1 - Valores médios de intensidade, frequência e contribuição dos EET-I, EET-II, EET-III obtidos na área da Bacia Amazônica

\begin{tabular}{|c|c|c|c|}
\hline Classe & $\begin{array}{c}\text { Média } \\
(\mathrm{mm})\end{array}$ & $\begin{array}{c}\text { Frequência } \\
\left(\mathrm{n}^{\text {o }} \text { de casos }\right)\end{array}$ & $\begin{array}{c}\text { Contribuição } \\
(\%)\end{array}$ \\
EET-I & 34,6 & 228 & 23,2 \\
EET-II & 55,9 & 65 & 9,7 \\
\hline EET-III & 85,1 & 32 & 7,0 \\
\hline
\end{tabular}

\section{Conclusões}

Neste trabalho foi feito a identificação de eventos extremos de chuva baseado na intensidade da precipitação com o intuito de determinar e classificar a frequência e a climatologia dos eventos extremos de chuva para os meses de DJF no período de 1998 a 2013. Os resultados mostram que os três tipos de eventos extremos de chuva ocorrem sobre toda a região amazônica, apresentando valores médios máximos sobre o oeste, sudoeste e sudeste do Estado do Amazonas e nordeste dos Estados do Pará e Amapá, com valores mínimos sobre o Estado de Roraima. Nota-se na frequência dos eventos extremos de chuva que as três classificações não apresentam valores bem distribuídos sobre a região de estudo. Nota-se que quanto menor (maior) é a frequência de um determinado tipo de evento maior (menor) é a intensidade do mesmo em produzir chuva. Por exemplo, os EET-I que apresentaram uma frequência aproximada de 80 casos, produziram em média $40 \mathrm{~mm}$ de chuva, enquanto que os eventos extremos de maior intensidade, EET-III, produziram em média $110 \mathrm{~mm}$ de chuva em aproximadamente 14 casos observados no período.

A contribuição dos EET-I, EET-II e EET-III no total de precipitação, mostrou que, na região Amazônica, ambas as classificações apresentam menor contribuição em uma faixa que vai do leste do estado do Amazonas até o Mato Grosso. De forma que, no extremo norte da região (norte do Estado do Amazonas, noroeste do Pará, Roraima e Amapá), os eventos extremos produzem maiores quantidades de chuvas. Assim, dos três tipos de eventos extremos de chuva, a maior contribuição no total de chuva é dos EET-I seguidos dos EET-II e EET-III. De forma que os EET-III contribuíram com 30\% da chuva no nordeste de Roraima.

\section{Referência}

BRITO, A. L.; Um estudo sobre a intensidade e frequência dos eventos extremos de chuva na Amazônia. Adriane Lima Brito (2012). Disponível em: <https://docs.google. com/file/d/0B3mU3tt-XAByd01NWFJEaTgzaEE/ edit?usp=drive_web\&urp=http://scientificmet.wordpress. com/material-didati\&pli=1>. Acesso em: 11 de julho de 2013.

CARVALHO, L. M. V.; JONES, C.; LIEBMANN, B. The South Atlantic Convergence Zone: persistence, intensity, form, extreme precipitation and relationships with intraseasonal activity. Journal Climate, v. 17, p. 88-108, 2003.

FRICH, C.; ALEXANDER, L. V.; DELLA-MARTA, P.; GLEASON, B.; HAYLOCK, M.; KLEIN TANK, A. M. G.; PETERSON, T. Observed coherent changes in climatic extreme during the second half of the twentieth century. Climate Research, v. 19, p. 193-212, 2002.

GAO, X.; PAL, J. S.; GIORGI, F. Projected changes in mean and extreme precipitation over the Mediterranean region from a high resolution double nested RCM simulation. Geophysical Research Letters, v. 33, 2006.

HEGERL, G. C.; ZWIERS, F.W.; STOTT, P.A.; KHARIN, V.V. Detectability of anthropogenic changes in anual temperature and precipitation extremes. Journal of Climate, v. 17, p. 3683-3700, 2004.

MARENGO, J. A.; ALVES, L.; VALVERDE, M.; LABORBE, R.; ROCHA, R. Eventos extremos em cenários regionalizados de clima no Brasil e América do Sul para o 
Século XXI: projeções de clima futuro usando três modelos regionais. Ministério do Meio Ambiente, Secretaria de Biodiversidade e Florestas, Diretoria de Conservação da Biodiversidade, relatório 5, p. 08-62, 2007.

MARENGO, J. A. Impactos de extremos relacionados com o tempo e o clima - Impactos sociais e econômicos. Boletim do Grupo de Pesquisa em Mudanças Climáticas, n. 08, p. $1-5,2009$.

MARENGO, J.A.; NOBRE, C.A.; CHOU, S.C.; TOMASELLA, J.;SAMPAIO, G.; ALVES, L.M.; OBREGÓN, G.O.; SOARES, W.R.; BETTS, R.; KAY, G. Riscos das Mudanças Climáticas no Brasil. Análise Conjunta BrasilReino Unido Sobre os Impactos das Mudanças Climáticas e do Desmatamento na Amazônia, p. 2-56, 2011.

ROZANTE, J. R.; MOREIRA, D. S.; GONÇALES, L. G. G.; VILA, D. A. Combining TRMM and Surface Observation Precipitation: Technique and Validation Over South American. Instituto Nacional de Pesquisas Espaciais, v.1, 2009.

SAREWITZ, D.; PIELKE Jr. R.; BYERLY Jr. R. Prediction: Science, Decision Making, and the Future of Nature (Covelo, CA: Island Press): p. 405, 2000.

VALVERDE, M; MARENGO, J. R. Rainfall Extremes Events Climatology over the Amazon Basin. Proceedings of the Global Conference on Global Warming, p. 1-8, 2011.

VILA, D. A., GONCALVES, L. G. G., TOLL, D. L., \& ROZANTE, J. R. Statistical Evaluation of Combined Daily Gauge Observations and Rainfall Satellite Estimates over Continental South America. Journal of Hydrometeorology, v. 10, p. 533-543, 2009. 